\title{
Data Fusion Architecture for Maritime Surveillance
}

\author{
Ahmed Gad \\ Department of Electrical and Computer Engineering, \\ Royal Military College of Canada, \\ Kingston, Ontario, Canada, K7K 7B4 \\ ahmed.gad@rmc.ca
}

\author{
M. Farooq \\ Department of Electrical and Computer Engineering, \\ Royal Military College of Canada, \\ Kingston, Ontario, Canada, K7K 7B4 \\ farooq@rmc.ca
}

\begin{abstract}
Various multisensor data fusion architectures have been utilized to support the Maritime Surveillance (MS) in maritime tactical and strategic operations. The military tactical situation is mechanized through data fusion thus improving the quality of target tracking system. One of the major problems is that the surveillance area is generally large, hence making it difficult to arrive at a feasible data fusion architecture. The latter arises due to timing, accuracy, and different types of sensors and sensor platforms. In this paper, various data fusion architectures for MS are discussed. The proposed system interacts with the data fusion processes at different information levels. This architecture is employed to support the MS for a typical maritime tactical scenario. The proposed architecture has an acceptable performance.
\end{abstract}

Keywords: Data fusion, maritime surveillance, situation assessment, threat assessment, strategic defense.

\section{Introduction}

Multisensor data fusion is one of the effective approaches for solving different sets of problems having common characteristics. It uses the data from multiple sensors to perform inferences that may not be possible from a single sensor alone. Data from different sensors are combined using signal processing, pattern and image recognition, artificial intelligence, and information theory. Input data from sensors can be target positional data, such as angular data, range information, and identity.

Numerous multisensor data fusion architectures have been discussed in the literature [1-10]. A common feature of these architectures is that they incorporate multiple levels of data processing within the data fusion process. In 1980, the intelligence cycle [11], the Joint Directors of Laboratories (JDL) model [12,13], and the Boyd Control, also called the Observe, Orient, Decide, and Act (OODA) model have been introduced [14]. In 1990, the Dasarathy model [15] and the Waterfall model [16] have been developed. Recently, the Omnibus [17] and the extended OODA [18] have been proposed.

Situation assessment establishes a view of enemy activities, events, locations, and maneuvers. Threat assessment is different from situation assessment as it estimates the degree with which engagement events may occur. Many authors have provided various overviews of the situation and threat assessment problems [19-25].

In this paper, the main existing data fusion architectures are reviewed. Then, a new model which may be regarded as the combination of the current models is proposed. The proposed data fusion model is then employed in the defense and surveillance areas. Defense applications include maritime surveillance, airto-air and surface-to-air defense, strategic warning and defense, and battlefield intelligence.

Maritime surveillance requires target detection, tracking, and identification. The identification includes target's identity, events, and activities. The activities of interest may be the launch of a missile from a ship, submarine, or ground based missile battery. The maritime surveillance area includes surface, subsurface, and airborne targets in a considerable large area. A large number of ships, submarines, and aircrafts may be involved in the surveillance process.

\section{Data Fusion Architectures Overview}

In the maritime surveillance environment, multiple observations and existing knowledge are employed using data fusion. In this section, we will review the existing data fusion models.

\subsection{The Intelligence Cycle [11]}

The intelligence process can be described as a cycle that can be used for modeling the data fusion process. The intelligence cycle comprises four phases; collection, collation, evaluation, and dissemination. In the collection phase, raw intelligence data are obtained. The sources are electronic sensors or human derived sources. The data is presented in the form of an intelligence report which has a high abstraction level. In the collation phase, the associated intelligence reports are analyzed, compared, and correlated. In the evaluation phase, the collated intelligence reports are fused and analyzed. In the dissemination phase, the fused intelligence is distributed to military commanders who use the information to produce the required decisions. 


\subsection{The JDL Model $[12,13]$}

The JDL model has been proposed by the US Joint Directors of Laboratories Data Fusion Sub-Group in 1985. The JDL model is divided into five levels. At level 0 , the pre-detection activities such as pixel or signal processing, spatial or temporal registration is present. Level 1 is concerned with estimation and prediction of target location, behavior or identity. Level 2 investigates the relations among entities such as force structure and communication roles. Level 3 delineates sets of possible courses of action and the effect on the current situation. Level 4 is an element of Resource Management used to close the loop by re-tasking resources to support the objectives of the mission.

The JDL model is not intended to prescribe a strict ordering on the data fusion levels. This was indicated diagrammatically by the use of an information bus rather than a flow structure. Nevertheless, data fusion system designers have consistently assumed this ordering. Clearly, there is a need from users to have an ordering while the authors of the JDL model rightly defend the need for a model which admits systems with different hierarchies at different levels.

\subsection{The Boyd Control Loop [14]}

The Boyd control loop (or OODA) loop is divided into 4 phases. The observe phase is comparable to the JDL Level 0 and part of the collection phase of the intelligence cycle. The orient phase includes the functions of JDL Levels 1, 2 and 3. It also includes the structured elements of collection and the collation phases of the intelligence cycle. The decide phase includes JDL Level 4 and the dissemination activities of the intelligence cycle. The act phase has no direct analogue in the JDL model and is the only model that explicitly closes the loop by taking into account the effect of decisions in the real world.

\subsection{The Waterfall Model [15]}

The Waterfall focuses on the processing functions at the lower levels. The sensing and signal processing levels correspond to JDL level 0 . The feature extraction and pattern processing levels correspond to to JDL level 1. The situation assessment level corresponds to JDL level 2 The decision making level corresponds to JDL level 3. The major limitation of the Waterfall model is that the feedback is not explicitly depicted.

\subsection{The Dasarathy Model [16]}

The Dasarathy model is based on fusion functions rather than tasks and may therefore be incorporated in each of the fusion activities. Many researchers have identified the three main levels of abstraction during the data fusion process as decisions (symbols or belief values), features (intermediate-level information), and data (more specifically sensor data). As was pointed out by Dasarathy, fusion may occur both within these levels and as a means of transforming between them.

\subsection{The Omnibus Model [17]}

The Omnibus model overcomes some of the main limitations of the previous models while capitalizing on their advantages. In the Omnibus model, feedback is explicit and the previously neglected concept of loops within loops is acknowledged. The cyclic nature of the data fusion process is made explicit by retaining the general structure of the Boyd loop. The fidelity of representation expressed by the Waterfall model is then easily incorporated into each of the four main process tasks. The levels in the process where fusion may take place are explicitly indicated.

\subsection{The Extended OODA Model [18]}

The Extended OODA model provides a mechanism for multiple concurrent and potentially interacting data fusion processes. It is decomposed into a meaningful set of $\mathrm{N}$ high-level functions. These functions are examined in terms of the Observe, Orient, Decide, and Act decision loop that constitute the OODA nodel. Each function can be further decomposed and evaluated with respect to each OODA phase. The extended OODA model is consistent with the OODA model in the high level as it closes the loop between the decision making and its environment. It is also consistent with the increasing level of abstraction for information processing in each level of the JDL model. It also provides the "loop within the loop" capability of the Omnibus model.

\section{Multisensor Data Fusion Application to Maritime Surveillance}

Many benefits can be earned when using multisensors data fusion in multitarget surveillance systems. The data from each sensor can be used to complement the data of the other sensors to obtain broader coverage and more accurate target estimates and ID decisions and in turn eliminate the false tracks and the countermeasures. The tracking accuracy can also be improved using similar sensor types such as distributed infrared sensors or radars for position estimates.

The actual deployment of the automated multiple sensor systems is very difficult since we have to accurately choose the proper tracking architecture to get more accurate surveillance pic ture than what we can get using the manual integration of data from the individual sensors. The proper tracking architecture must consider important issues such as time alignment and synchronization of tracks and measurements, the 
resolution of the sensors, data association using dissimilar nature data, and the registration problem. The multisensor data fusion has been widely used in the last decade in the military application. The military defense applications include the air-to-air and surface-to-air defense, strategic warning and defense, battlefield intelligence, and maritime surveillance.

For the air-to-air and surface-to-air defense, it is necessary to detect, track, an identify aircraft at proper ranges so that the defense weapons would have enough time to respond to the threats. In the strategic warning and defense scenario, the aim is to detect the indications of the strategic actions and to be able to detect and track ballistic missiles and warheads and to detect the enemy's military activities such the enemy's force distribution, movements, and intention. In the battlefield intelligence, the aim is to detect and identify ground targets to get a better idea about the enemy's force, tactics, and strategies. This system utilizes the enemy order of battle and creates the enemy database. This database should include the identification, location, and characteristics of the enemy's military units. This helps us to determine the enemy's intent and situation and we will be able to calculate the degree of threat.

In the maritime surveillance area, the sensors could be co-located, distributed, or combinations of co-located and distributed. The co-located sensors may be RADAR, LADAR, IR, EO, and ESM located on the satellite, aircraft or ship (i.e. mobile platforms). The distributed sensors may be a number of radars with different coverage ranges and regions. The fusion process may have different sensors that produce dissimilar outputs such as radar and imaging infrared sensors. The design and analysis of distributed systems is not easy when only angle measurement data are available. Similarly, the passive ESM sensors produce only target identification but can not provide range measurement. Thus, a wide variety of sensors and configurations can be used in the multisensor environment. A Typical scenario of the maritime surveillance environment is shown in figure 1 .

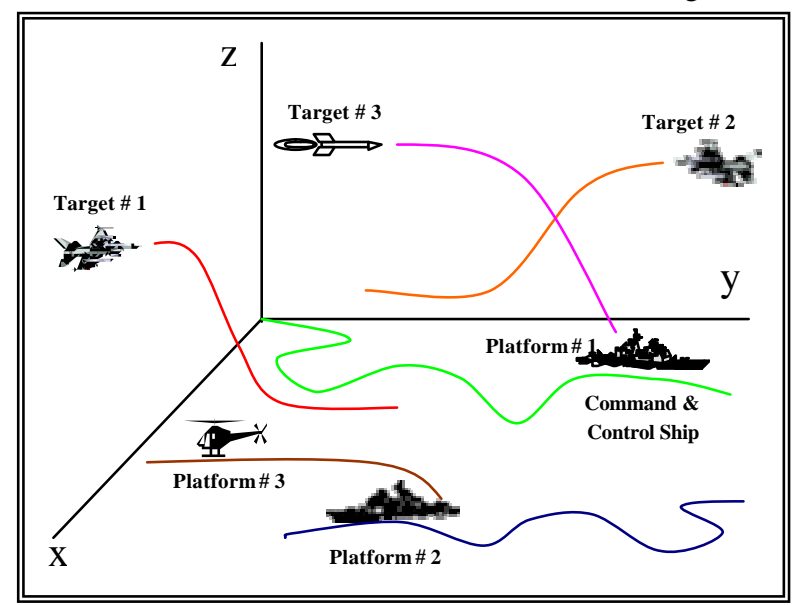

Fig. 1 Typical Maritime Surveillance Scenario [26]
As shown in figure 1, three different platforms are involved in the surveillance process; two ships and one helicopter. Three aerial targets are presented, two fighters and one missile. Radars, infrared, ESM, and laser radar sensors are distributed onboard ships and helicopters. The multiple observations of positional, activity, intent, and identity data are combined to determine an estimate of the targets behavior.

In this paper, modern data fusion architecture is developed, which includes the data collection level where the sensors are used while distributed on different platforms. The second level processing, called low-level processing includes the data association, data alignment, sensor registration, and identity and position fusion. The high-level processing includes the Situation Assessment (SA), threat assessment, and sensor management. The general proposed architecture is shown in Figure 2.

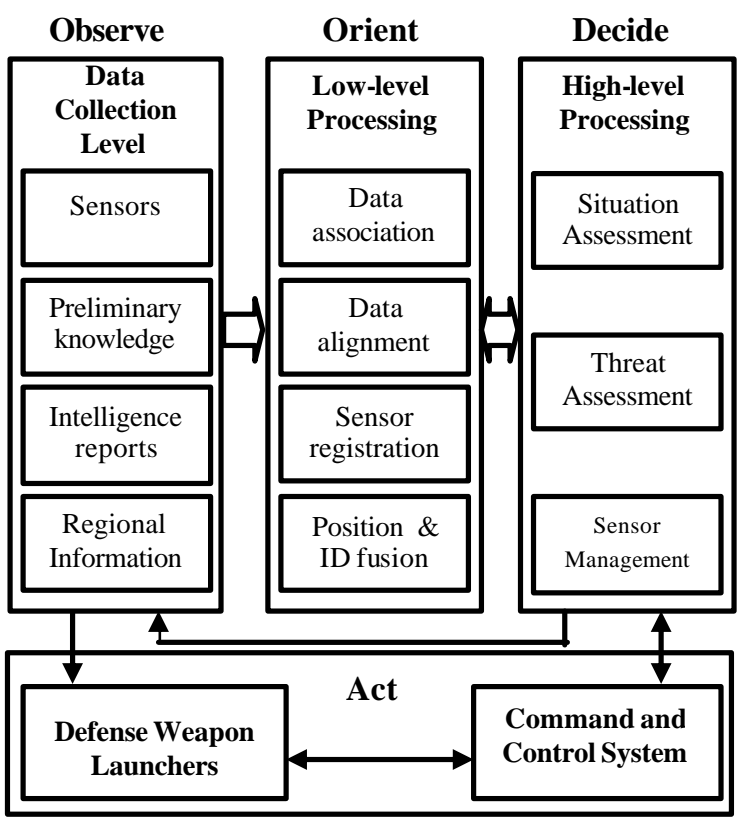

Fig. 2 The proposed target tracking architecture

The proposed target tracking architecture interacts with the data at different processing levels as shown in figure 8. It is considered as a combination of the existing data fusion architectures as it has the advantage of data ordering presented by the JDL model, the act phase added by the OODA loop, the loop-in-the-loop introduced by the Omnibus model, and finally the function level processing presented by the extended OODA model.

\subsection{Data collection level processing}

The data collection level is comparable to the JDL level 0 and the Boyd control loop Observe phase. Clearly, the environment includes different sensors and platforms which make the tracking problem more 
complicated. These complications are due to the complexity of arriving at perfect communication links between the platforms and the fusion center as well, the data collected from the sensors are of different nature and so the fusion process should consider this difference. However, using the different information enables us to draw a considerably complete picture of the surveillance area. The preliminary knowledge data base and the regional information (weather, the nearest coastal defense bases, sensors coverage information, weapon coverage information, etc.) are included in the data collection level. The intelligence reports are also useful to get better knowledge about the force distribution, intent, and activities of the enemy.

The output of this level is the targets data which are collected from different sources, namely IR, IFF, ESM, LADAR, and Radar. These data are the position, attribute, activities, and intension of the targets and are collected using three different platforms, command and control ship (platform \#1), another ship (platform \#2), and helicopter (platform \#3) which is a part of platform \# 2 and can be co-located onboard the ship to reduce the number of platforms to two instead of three. The collected data contains the information about 3 aerial targets, fighter (platform \#1), fighter (platform \#2), and attacking missile (platform \#3), as shown in figure 1.

\subsection{Low-level data processing}

The low-level data processing involves the data association issue, the data alignment problem, the sensor registration problem, and the position \& ID fusion. The low-level data processing is comparable to JDL levels 1 , and 3 and the Boyd control loop Orient phase.

\section{2.a Data Association}

Data association is the way to correlate observations from multiple sensors into data groups each related to a single target. The problem is difficult in a dense tracking environment where we have many close targets of unknown identities. Data association compares the observations and determines which observations belong to what target. The main objective is to associate in a correct fashion such that we correlate every measurement to the target that it comes from. Due to the uncertainty of measurement origin because of the noise or clutter, the performance measure of the whole tracking system will depend mainly on the data association. Conventionally, several tracking algorithms have proposed ranging from the nearest neighbor to the probabilistic data association technique. Recently, the knowledge-based systems have been employed to solve the data association problem [27-31]. These knowledgebased systems include fuzzy logic, neural networks, artificial intelligence, and Viterbi algorithms.

\section{2.b Data Alignment}

Data alignment transforms data received from multiple sensors into a common spatial and temporal reference frame. Alignment functions include coordinate, time, and unit conversions. Data alignment is very important issue as the measurements are collected using different platforms. The coordinate alignment problem is solved by using the global coordinate system instead of using the local coordinate frame of each platform. The data here are transformed and used in the polar coordinate system as the IR, ESM sensors provide only angle information and the LADAR and radar provide both the angle and range information. The time alignment problem could be solved by synchronizing the platforms and with the global fusion node. However, due to the different sampling period of the sensors used, the measured time is asynchronous and time alignment is still needed. The Least Squares Method can be used to derive the time alignment expression of the sensors, [32].

\section{2.c Sensor Registration}

Sensor registration is one of the main problems that can affect the performance of the overall tracking system. The registration error must be corrected to be able to estimate and correct the sensor measurement biases so that the multiple sensor measurements and tracks can be referenced to a common coordinate system. The registration error can cause the formation of ghosts. The most important sources of registration error are the misalignment of the measurement axes, the measurement bias error, and the sensor location error. Many registration methods have been discussed in the literature including the simple averaging method, cross correlation, matrix inversion, least-square, Kalman filtering, neural networks, parallel processing techniques, and artificial intelligence-based methods [9,33-40].

\section{2.d Position Fusion}

The sensor fusion problem requires the development of methods to combine the data from multiple sensors for tracking and identification. The use of multiple sensors requires the fusion of different types of data such as sensor reports containing measured attributes (target type and feature).

The dual tracking and identification problem can be developed by performing data association based on kinematic quantities and then computing the target ID. In the proposed data fusion system, an alternative approach, shown in figure 3 , has been employed [41].

The composite kinematic/ID single-sensor multitarget (SSMT) tracker involves three main modules. In the gating module, the kinematic gating is performed first and then the attribute gating is performed. The data association module employs the 
attribute gating output in parallel with both the kinematic and attribute association. Then, the association outputs from kinematic and attribute are combined by determining the similar measures to provide an input to the estimation module. In the estimation module, the measurement-to-track assignment strategy is chosen first and then the kinematic and identity estimates are updated. From the output of the estimation module, the composite kinematic and identity tracks are fed back to the gating module to assess in screening the infeasible kinematic and attribute pairs.

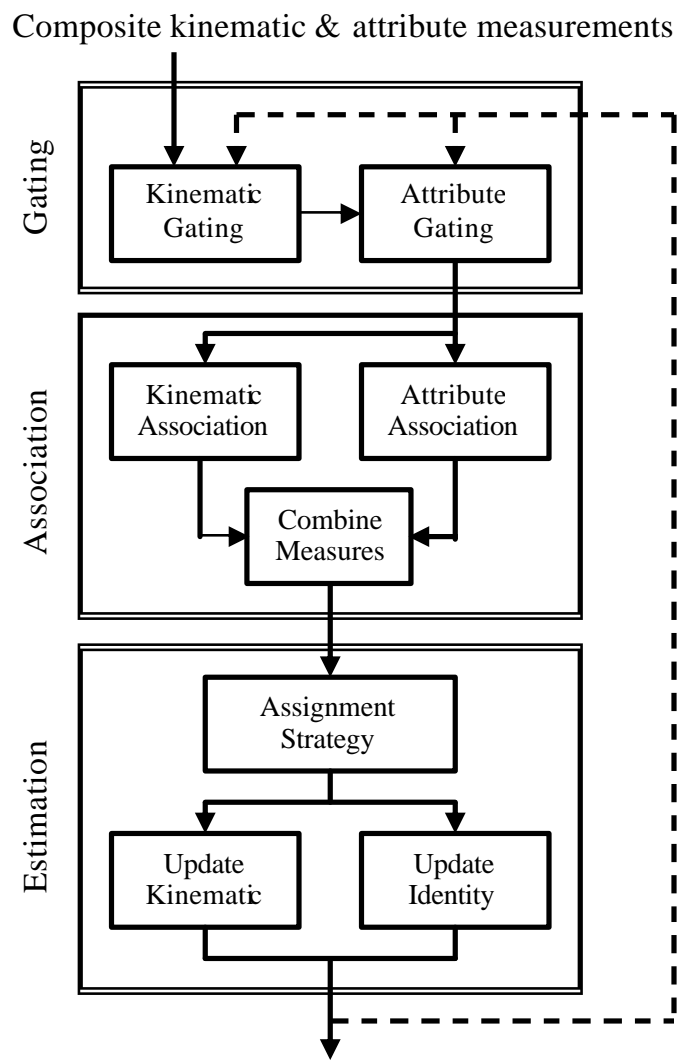

Composite kinematic and identity tracks

\section{Fig. 3 Composite kinematic/ID single-sensor multi- target (SSMT) tracker}

In a multisensor tracking system we must first define the level at which sensor data will be fused into tracks. There are three ways to combine the data: sensorlevel, central-level, or combined data fusion.

The sensor-level fusion is shown in figure 4. Each sensor will maintain its own track file. Then, the sensorlevel track files will be updated using communication links among the sensors and between the sensors and the central track file. The sensor-level tracks must be combined into a central track file. Thus, each sensor will have separate track files and a central track file will be formed as a composite of these files. The advantages of using sensor-level fusion are the reduced data-bus loading, the reduced computational loading, and the higher survivability due to distributed tracking capabilities. The disadvantages are due to the fact that the central-level tracks are updated with sensor-level track data, instead of sens or report data. If a central-level track is updated with a sensor-level track, the usual assumption of error independence from one update period to another is not valid.

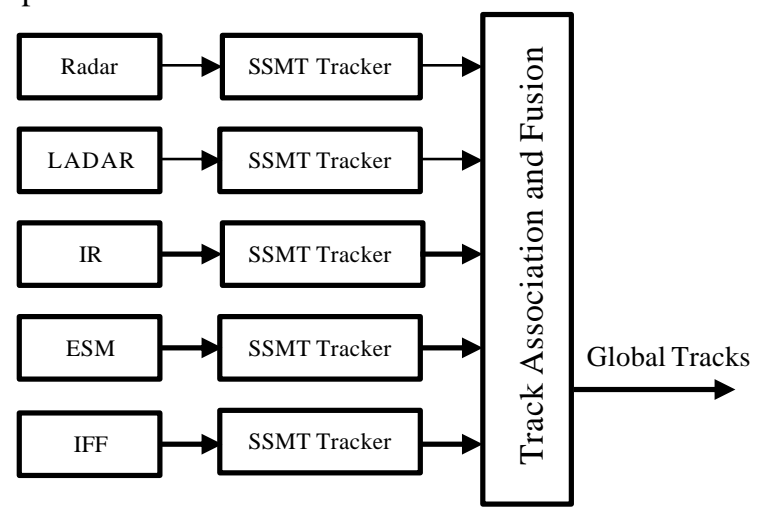

Fig. 4 Decentralized sensor-level data fusion

The second alternative for combining the sensor data is the central-level fusion shown in figure 5. In this approach, all the report data are sent directly to a central processor where a master track file is maintained. The advantage of this approach is the improved tracking accuracy because the target track is performed using the observations from more than one sensor and thus resulting in fewer miscorrelations. Another advantage is the improvement of track confirmation and continuity. The disadvantage of using central-level fusion is when one sensor becomes faulty it will affect the central-level tracking performance.

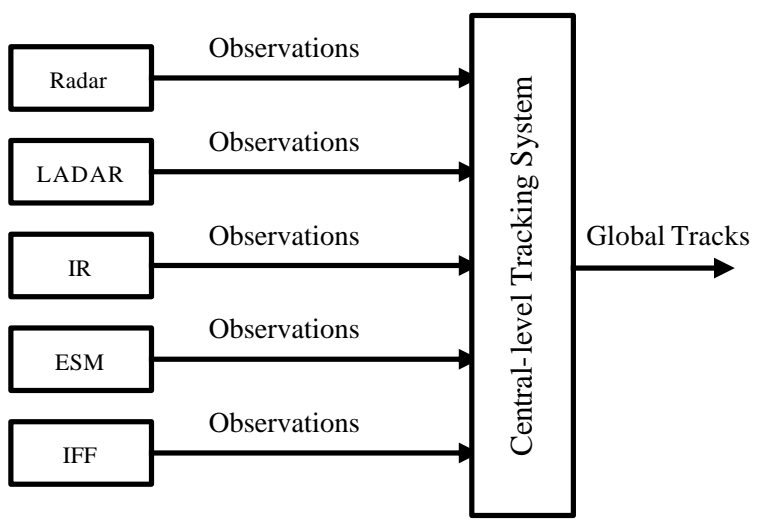

Fig. 5 Central-level data fusion

There are several other approaches which are intermediate between sensor-level and central-level tracking which is called hybrid or combined fusion. One method averages all the measurements from a given target for a certain time interval and uses this average to 
update the central track. Another method forms a central-level track from one of the sensors and then allows selected measurements from all sensors to update that central track.

In the real world, the problem forces a constraint of using specific fusion architecture over the others. Based on present work, it has been found that combined fusion architecture, shown in figure 6 , is a more efficient means of tracking the problems encountered in MS, [42].



Fig. 6 Combined Position Fusion Block diagram

\section{2.d Identification Fusion}

Identity fusion combines data related to identity such as actual declarations of identity from sensors or other parametric data. Identity fusion techniques include cluster methods, adaptive neural networks, templating methods, Dempster-Shafer, and Bayesian inference methods. The proposed ID fusion flow chart for one cycle is shown in figure 7 .

\subsection{High-level data processing}

The high-level data processing involves the situation assessment issue, the threat assessment issue, and the sensor management. The high-level data processing is comparable to JDL level 4 and the Boyd control loop Decide phase.

\section{3.a Situation Assessment}

The Situation assessment system provides information about enemy's activities, events, locations, and maneuvers. All these aspects will allow us to know what is happening or what is going to happen. The situation assessment is a considerably subjective system which yields to a high performance that can not be obtained with the same system under the sole authority of the command and control operator. This is due to the fact that the operator is only concerned with the ultimate control and not with the detailed control.

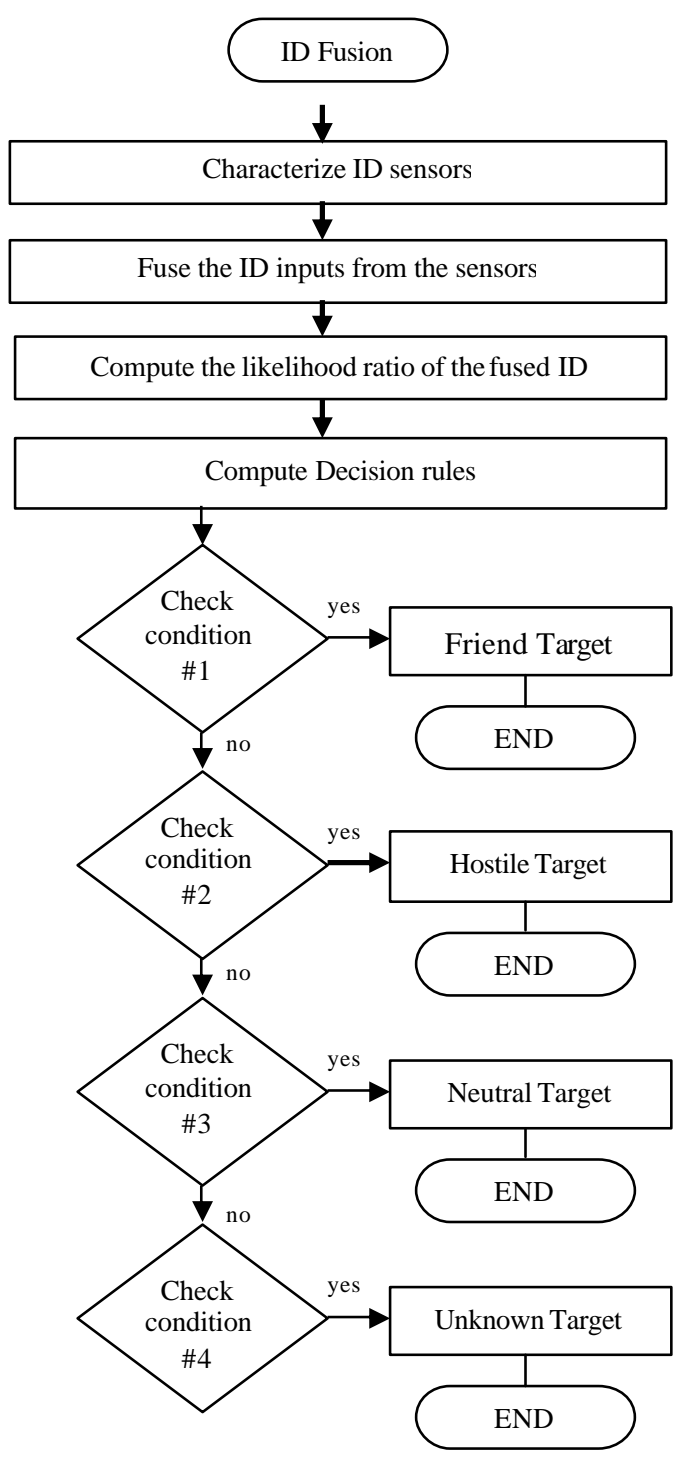

Fig. 7 ID Fusion Flow Chart

\section{3.b Threat Assessment}

Threat assessment is different from situation assessment as it estimates the degree with which engagement events may occur. Threat assessment determines the lethality and the compositional assessment of friendly and enemy forces. It also evaluates the indications and warnings of events, targeting calculations, and weapon assessment calculations. Many authors have provided various overviews of the situation and threat assessment problems, [43-44]. The block diagram of the situation and threat assessment model is shown in figure 8 . 


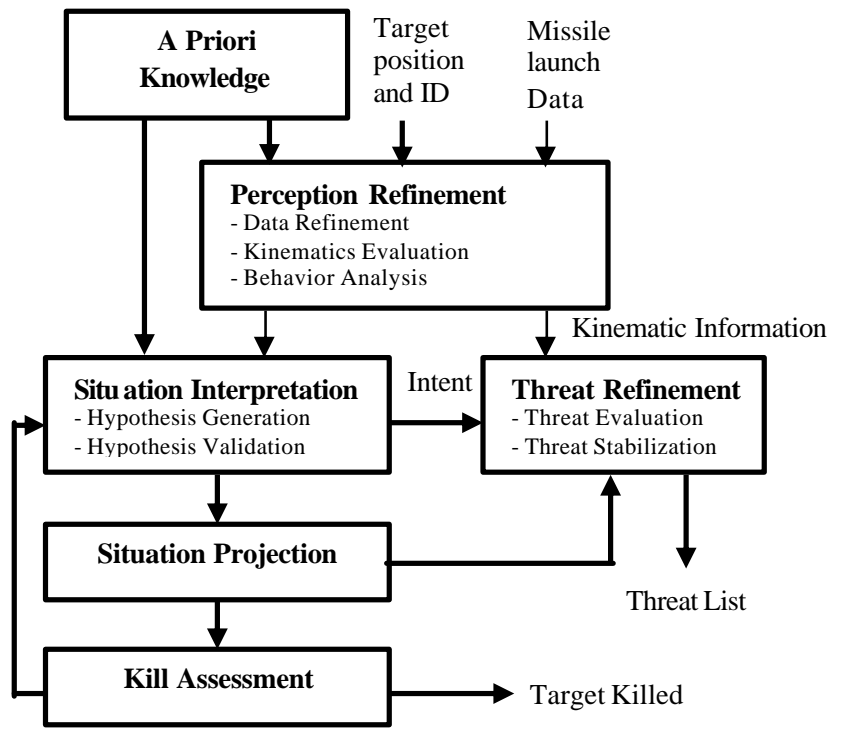

Figure 8 Situation and Threat Assessment Model

\section{3.c Sensor Management}

The sensor management is employed to optimize the use of sensor resources by adapting their use to the present tactical situation. The sensor management system should be responsive and adaptive to changes in the environment and the changing goals of its platform. This system produces a number of demanding tasks such as observation prediction for each sensor type and entity in the track database. Sensor management schedules sensors to ensure observational coverage of activities of interest and also monitors the performance and status of all sensors in the system.

\subsection{The Act Phase}

At the command and control system, the situation and threat assessment data are collected, based on this data the command and control system assign the suitable weapon and send the fire orders to the defense weapon launchers. These launchers are distributed on platforms in remote locations such as a coastal defense missile battery or a fighter outside the surveillance area.

\section{Conclusion}

In this paper, the maritime tactical situation is mechanized through data fusion architecture. This architecture improves the quality of the overall target tracking system. The proposed data fusion system considers the data alignment problem thus improving the timing problem. It also utilizes the hybrid fusion architecture to improve the accuracy. The compositional kinematic/ID fusion is used in the sensor level processing. The ID fusion algorithm is developed to assess the threat and perform a situation assessment. The proposed system interacts with the data fusion processes at different information levels. This architecture is employed to support the MS for a typical maritime tactical scenario.

The large-scale maritime surveillance system involves the multiprocessing between distributed data, external interfaces to real-time sensors, mobile platform constraints, and reliability requirements. It also involves data fusion systems which include the fusion algorithm selection, proper timing to fuse data, and man-in-theloop role. Future work is directed towards adding two issues to the fusion testbed. Firstly, man-in-the-loop which assess in the decision-making process based on the situation and threat assessment measures. Secondly, the model selection module which will allow the choice of the suitable association technique based on the maritime situation measures.

\section{References}

[1] S. Blackman, "Multiple-Target Tracking with Radar Applications", Artech House, 1986.

[2] C. Weaver, "Sensor Fusion", Proceedings of SPIE, Vol. 931, Orlando, FL, April 1988.

[3] P. Schenker, "Sensor Fusion, Spatial Reasoning and Scene Interpretation", Proceeding of SPIE, Vol. 1003, Nov. 1988.

[4] J. Llinas and E. Waltz, "Multisensor Data Fusion", Artech House, 1990.

[5] D. Hall and R. Linn, "A Taxonomy of Multi-Sensor Data Fusion Techniques", Proceeding of the 1990 Joint Service Data Fusion Symposium, Vol. 1, pp. 593-610, May 1990.

[6] S. Blackman and T. Broida, "Multiple Sensor Data Association and Fusion in Aerospace Applications", J. Robotic Systems, Vol. 7, pp. 445-485, June 1990.

[7] D. Hall, "Mathematical Techniques in Multisensor Data Fusion", Artech House, 1992.

[8] R. Antony, "Principles of Data Fusion Automation", Artech House, 1995.

[9] S. Blackman and R. Popoli, "Design and Analysis of Modern Tracking Systems", Artech House, 1999.

[10] Y. Bar-Shalom and W. Blair, "MultitargetMultisensor Tracking: Applications and Advances Volume III", Artech House, 2000.

[11] A. Shulsky, "Silent Warfare: Understanding the World of Intelligence", Brassey's, 1993.

[12] F. White, "A Model for Data Fusion", Proc. of the $1^{\text {st }}$ Int. Symp osium on Sensor Fusion, 1988.

[13] A. Steinberg, C. Bowman, and F. White, "Revisions to the JDL Data Fusion Model", SPIE, Vol. 3719, pp. 430-441, 1999.

[14] J. Boyd, "A Discourse on Winning and Losing", Maxwell AFB Lecture, 1987.

[15] B. Dasarathy, "Sensor Fusion Potential Exploitation - Innovative Architectures and Illustrative 
Applications", IEEE Proceeding, Vol. 85, No. 1, pp. 24-38, 1997.

[16] M. Bedworth, "Probability Moderation for Multilevel Information Processing”, DRA Technical Report DRA / CIS(SEI) / 651 / 8 / M94.AS03BPO32 / 1, 1994.

[17] M. Bedworth and J. O'Brien, "The Omnibus Model: A New Model of Data Fusion?", Proceeding IEEE AES Systems Magazine, pp. 30-36, April 2000.

[18] E. Shahabzian, D. Blodgett, and P. Labbe, "The Extended OODA Model for Data Fusion Systems", Fusion'2001, pp. FrB1-19-25, Aug. 2001.

[19] D. Noble, "Template-Based Data Fusion for Situation Assessment", 1987 Tri-Service Data Fusion Symposium. Johns Hopkins University, pp. 156-161, June 1987.

[20] M. Vineberg, "A Proposed User-Centric Battle Management System", Proceeding of the 12th Ship Control Systems Symposium, Oct. 1999.

[21] D. Ballard and L. Ripley, "A Knowledge-Based Decision Aid for Enhanced Situational Awareness", Proceeding of IEEE DASC 14th, pp. 340-347, 1994.

[22] J. Molina, F. Jimenez, and J. Casar, "Symbolic Processing fro Coordinated Task Management in Multiradar Surveillance Networks", Fusion'98, pp. 725-732, July 1998.

[23] I. Dall, "Threat Assessment without Situation Assessment", Proceeding Information, Decision, and Control (IDC'99), pp. 365-370, Feb. 1999.

[24] J. Ross, “An Instructible Agent to Assist Man-InThe-Loop Threat Assessment", Fusion'2001, pp. ThC3-25-32, Aug. 2001.

[25] S. Jameson, "Architectures for Distributed Information Fusion to Support Awareness on the Digital Battlefield", Fusion'2001, pp. FrB1-27-35, Aug. 2001.

[26] R. Nedvidek, M. Johnson, L. deKok, and M. Farooq, "A Prototype for Sensor Data Fusion", Nato AC/243 (panel 3) and (panel 4), Symposium on Multi-Sensor and Sensor Data Fusion, Session 3, Paper \#3.1.9, pp. 21-1 to 21-20, vol.2, Brussels, Belgium, Nov. 1993.

[27] T. Quach and M. Farooq, "A Fuzzy Logic-Based Target Tracking Algorithm," SPIE, vol. 3390, pp. 476-487, April 1998.

[28] H. Osman, M. Farooq, and T. Quach, "Fuzzy Logic Approach to Data Association," SPIE, vol. 2755, pp. 313-321, April 1996.

[29] A. Gad, M. Farooq and S. Midwood, “Applications of Fuzzy Logic to Target Tracking in a Cluttered Environment", SPIE, vol. 4380, pp. 91-104, April 2001.

[30] A. Ashraf, M. Tummala, R. Cristi, "Fuzzy logic data correlation approach in multisensor-multitarget tracking systems", Signal Processing 76, pp. 195209, 1999.
[31] C. Looney and Y. Varol, "Fuzzy Clustering for Association and Fusion in Multitarget Tracking with Multisensors", Fusion'99, vol. 1, pp. 255-261, July 1999.

[32] X. Wang, Z. Jing, F. Chen, and X. Yao, "ActivePassive Joint Tracking with Infrared and Laser Sensors", Proc. CDC, Orlando, FL, Dec. 2001.

[33] M. Dana, "Registration: A Prerequisite for Multiple Sensor Tracking”, Multitarget-Multisensor Tracking: Advanced Applications, Y. Bar-Shalom (Ed.), Artech House, Ch. 5, 1990.

[34] H. Blom et al., "Design of a Multisensor Tracking System for Advanced Air Traffic Control", Multitarget-Multisensor Tracking: Applications and Advances, Vol. II, Y. Bar-Shalom, Ed., Artech House, Chapter 2, 1990.

[35] Abbas, H., et al., "Track-Independent Estimation Schemes for Registration in a Network of Sensors", Proc. CDC, pp. 2563-2568, Dec. 1996.

[36] N. Nabaa and R. Bishop, "A Recursive Solution to the Sensor Registration Problem in a Multiple Sensor Tracking Scenario", SPIE, Vol. 2759, pp. 385-396, April 1996.

[37] D. Crowley and B. Shafei, "Registration in MultiSensor Data Fusion and Tracking", Proc. ACC., pp. 875-879, June 1993.

[38] S. Dhar, "Application of a Recursive Method for Registration Error Correction in Tracking with Multiple Sensors", Proc. ACC., pp. 875-879, June 1993.

[39] H. Leung et al., "Comparison of Registration Error Correction Techniques for Air Surveillance Radar Network', SPIE, Vol. 2561, pp. 498-508, July 1995.

[40] R. Kenefic, "Local and Remote Track File Registration Using Minimum Description Length", IEEE Trans. on AES, Vol. AES-29, No. 3, pp. 651655, July 1993.

[41] S. Bruder, R. Johnson, and M. Farooq, "Coordinated Multiple Sensor Multiple Target Tracking and Identification", Dept. of Elec. \& Computer Eng., Royal Military College of Canada, Tech. Report No. 94/6, May 1994.

[42] S. Bruder and M. Farooq, "Sensor Fusion Techniques and A Hybrid Multi-Sensor MultiTarget Tracker", Dept. of Elec. \& Computer Eng., Royal Military College of Canada, Tech. Report No. 90/3, Sept. 1990.

[43] D. Noble, "Managing Temporal Uncertainty in situation assessment", Proc. Of the 1988 Tri-service Data Fusion Symp ., vol. 1, May 1988.

[44] I. Dall, "Threat assessment without situation assessment", In proc. Information, Decision and Control (IDC'99), pp. 365-370, Feb. 1999. 